\title{
Self-Organizing Map (SOM) in Wind Speed Forecasting: A New Approach in Computational Intelligence (CI) Forecasting Methods
}

\author{
Mohammad Amin Esmaeili \\ Faculty: Janet Twomey \\ Department of Industrial and Manufacturing Engineering
}

Abstract. Two critical issues in renewable energy are how to make wind energy cost effective and how to integrate wind energy into electricity grids. Within many approaches to cost reduction, wind speed forecasting was mentioned as an effective approach because accurate forecasting of wind speed has a direct impact on the scheduling of a power system, and also the dynamic control of the wind turbine. This research investigates the practical use of Self Organizing Map (SOM) as a special type of neural network based forecasting method. In this paper, forecasting the average, maximum and minimum of one-day-ahead wind speed based on the past wind speed states of the previous 24 hours is the objective.

\section{Introduction}

Within approaches to make wind energy more cost effective, wind speed forecasting has been established as a method which can be used to significantly reduce the operating cost of wind energy (M“ohrlen, 2004). Based on Western Electricity Coordinating Council (WECC) results, the WECC operation cost impact of not using a forecast is $\$ 12$ 20/MWh and an additional \$1-2/MWh could be saved if these forecasts could be made perfect (Milligan, 1995). The reason is that operational cost of additional reserved generators, which are predicted for non-wind situations, can be reduced by a better wind speed forecasting system. In other words, accurate forecasting of wind speed is essential since it has a direct impact on scheduling of a power system, and the dynamic control of the wind turbine (Wu, 2007).

The forecasting methods of wind speed or any other time-series can be divided to two categories: (1) the statistical time-series forecasting methods like ARIMA or Bayesian method and (2) the computational intelligence (CI) forecasting methods like artificial neural network or fuzzy logic systems. The research presented here is applies the Kohonen Self Organizing Map (SOM) as a special type of neural network based forecasting method. This is a different approach since the SOM finds like clusters of like data and does not predict values given a set of inputs. The data set used for this study is based on 2 years of wind monitoring at the Beaumont site in Kansas that was performed $u$ under the Utility Wind Resource Assessment Program (UWRAP). Two subsets of data, which were gathered on 94 consecutive days of summer in 1997 and 62 days of spring in 1998, were identified as 'clean' data that can be used for our analysis; i.e. no missing values. The 94 days of data were chosen for training the proposed neural network and the 62 days of data for spring is used in the validation of the trained network.

\section{Initial data analysis and network inputs}

To find the best set of forecasting parameters, temperature, solar radiation and the numbers of hours wind speeds to include were investigated.

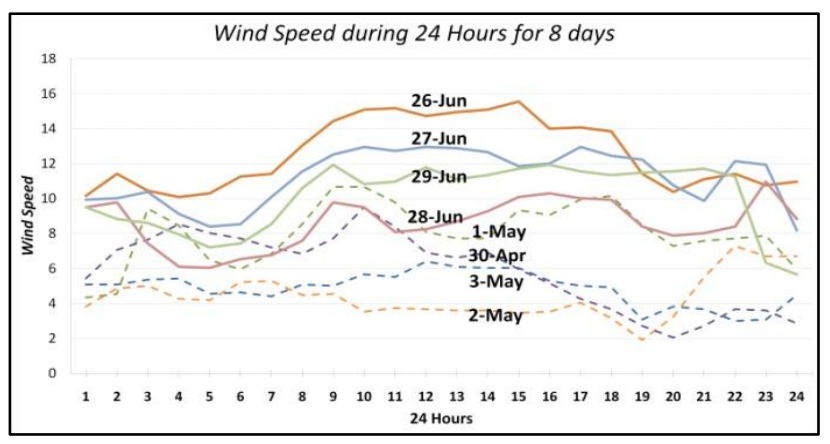

Figure 1: Wind speed for 2 four-day periods

Figure 1 is a plot of wind speeds over 24 hours. The dashed lines (four consecutive days of wind speeds) fluctuate around a constant mean which is different from the average of solid lines that represent 4 different consecutive days in June. The analysis and plot reveal that the potential variable for forecasting next day wind speeds is previous 24 hours wind speed data (24 inputs to SOM).

\section{SOM method for data clustering and wind speed forecasts}

The rational for using SOM for next day wind speed forecasts is as follows: given 24 hour wind speed input, the SOM will cluster like days. Like days will have similar next day (24 hour) wind speeds (next day average over 24 hours, and minimum and maximum over 24 hours). The training set is based on 94 days of wind speed data, with an input vector of 24 hours of wind speeds. The "Viscovery SOMine 5.0" software was used to create and train the network. The number of segmentations and training epoch were changed to create good results: 8 segments and 2000 epochs. The result was 8 clusters. The Viscovery builds a SOM Ward Cluster map (Figure 2), which is based on Ward's agglomerative hierarchical algorithms (Lattine \& Carroll, 2003). The Ward's algorithm automatically 
segments the map into interpretable groups of map units that correspond to clusters in the input data.

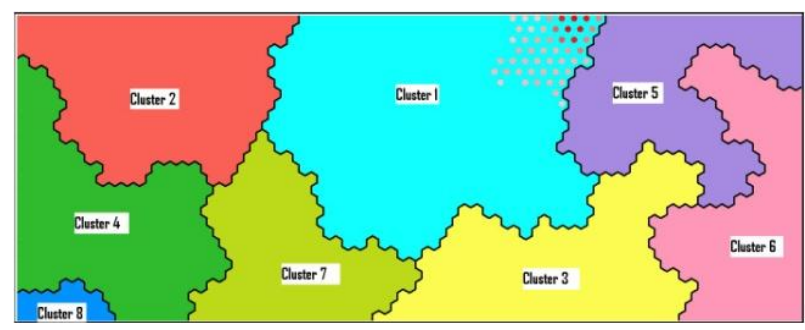

Figure 2: The SOM Ward Cluster map for 24 hours prediction

The average wind speeds over 24 hours for the 8 clustered wind speeds are shown in Figure 3. The next day minimum, average and maximum predicted values of wind speed for each cluster is given in Figure 4.

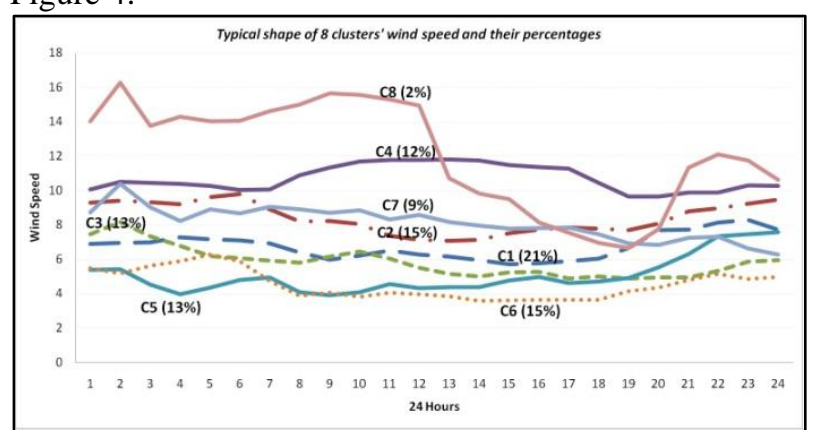

Figure 3: Shape of 8 wind speed clusters as an input of SOM

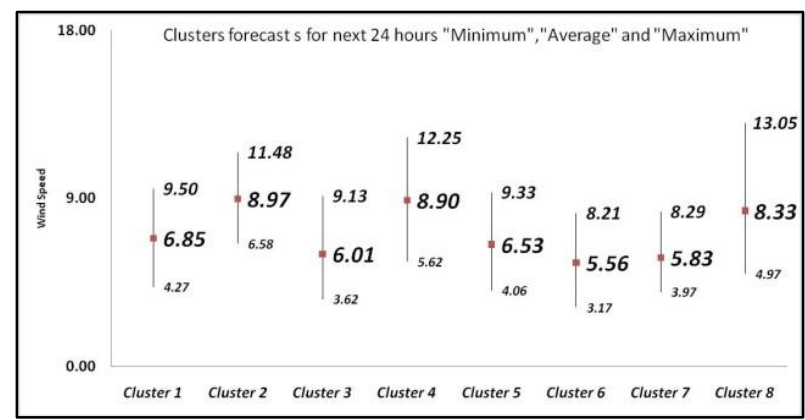

Figure 4: The forecasted wind speed parameters for the next 24 hours

\section{Testing the results}

To test the trained network 62 days in 1998 was used. A t-test was performed to detect the difference in forecasted values form the actual values. One cluster did not have sufficient number of samples to per from the test. The t-test at $\alpha=0.05$ indicate no statistical evidence to reject the equality of the sample mean and forecast mean for seven clusters. This fact allows us to use the forecast result for predicting the average wind speed of the next day based on the state of today's wind speed. Figure 5 and Figure 6 shows the test results for one of the 8 clusters.

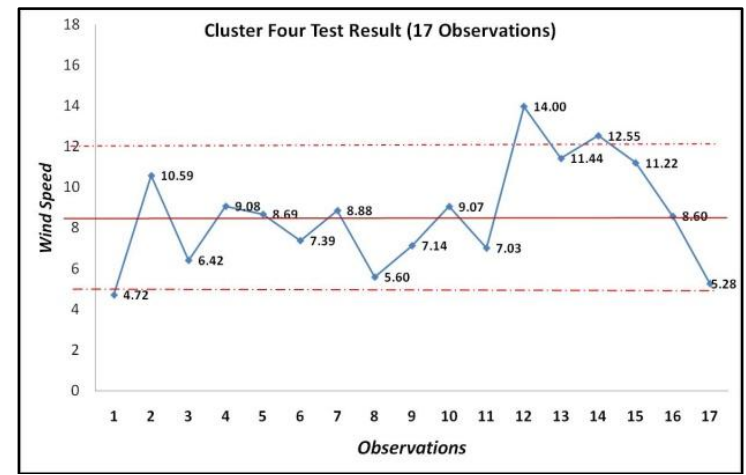

Figure 5: Cluster 4 forecast's mean and 95\% C.I. and 17 test data

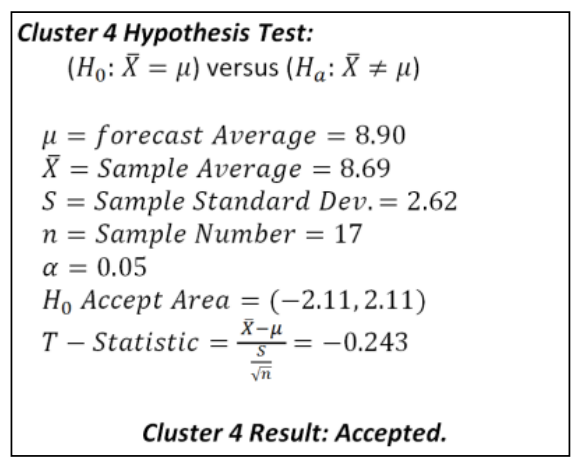

Figure 6: The hypothesis test detail for cluster 4 results

\section{Conclusions}

Wind speed forecasting is important in driving down the costs. A SOM neural network approach for wind speed forecasting was presented. The results indicate that SOM gives reasonably accurate next day average wind speed forecasts. This was demonstrated by testing the trained SOM using 62 days of data not used in training. The network forecasts of average wind speed were accurate in 7 clusters.

\section{References}

[1] Lattine \& Carroll. (2003). Cluster Analysis, Analysing Multivariate Data. Pacific Grove: Thomson Brooks/Cole.

[2] M"ohrlen, C. (2004). Uncertainty in Wind Energy Forecasting, PhD dissertation. University College, National University of Ireland.

[3] Milligan, M. (1995). Estimating the Economics Value of Wind Forecasting to Utilities. Golden, Colorado: National Renewable Energy Laboratory.

[4] Wu, Y. K. (2007). A literature review of wind forecasting technology in the world. Taiwan National Science Council. 